\title{
Um estudo sobre as ondas de choque no meio interplanetário
}

\author{
Vânia Fátima Andrioli ${ }^{(1,2)}$, Jairo Francisco Savian ${ }^{(1,2)}$, Ezequiel Echer ${ }^{(3,4)}$, Nelson Jorge Schuch ${ }^{(1)}$.
}

(1) Instituto Nacional de Pesquisas Espaciais - Centro Regional Sul de Pesquisas Espaciais - Santa Maria - RS, Brasil;

(2) Universidade Federal de Santa Maria - Laboratório de Ciências Espaciais de Santa Maria - Santa Maria, RS, Brasil;

(3) Instituto Nacional de Pesquisas Espaciais - Divisão de Geofísica Espacial (permanente) - São José dos Campos - SP, Brasil;

${ }^{(4)}$ Max Planck Institut fur Sonnesystemforschung (temporary), Katlenburg-Lindau, Germany.

(vania@lacesm.ufsm.br/ Fax: +55 - 55220 8007)

Copyright 2005, SBGf - Sociedade Brasileira de Geofísica

This paper was prepared for presentation at the $9^{\text {th }}$ International Congress of the Brazilian Geophysical Society held in Salvador, Brazil, 11-14 September 2005. Contents of this paper were reviewed by the Technical Committee of the $9^{\text {th }}$ International Congress of the Brazilian Geophysical Society. Ideas and concepts of the text are authors' responsibility and do not necessarily represent any position of the SBGf, its officers or members. Electronic reproduction or storage of any part of this paper for commercial purposes without the written consent of the Brazilian Geophysical Society is prohibited.

\section{Resumo}

O meio interplanetário, o espaço entre os planetas e o Sol, está permeado pelo vento solar que é a própria atmosfera solar se expandindo no espaço interplanetário. Esta atmosfera é composta por um plasma tênue magnetizado o qual favorece a ação das forças Coulombianas de longo alcance. Estas pela presença deste campo magnético, conseguem exercer seu papel que é o de transferência de momentum e informação às partículas provenientes do Sol. Qualquer mudança na atividade solar se reflete no meio interplanetário devido a transferência de informações. A agitação do Sol, conseqüência de sua constante atividade, é transmitida ao vento solar e freqüentemente produz Ondas de Choque. É possível, com o estudo das variações dos parâmetros interplanetários como: densidade, temperatura e velocidade do vento solar e intensidade do campo magnético interplanetário - $\mathrm{CMI}$, identificar a presença desses choques e calcular sua velocidade, intensidade e direção. Neste trabalho utilizamos dados de plasma e CMI fornecidos pelo sistema de dados On-line da sonda ACE - Advanced Composition Explorer, para fazer um estudo acerca de ondas de choque, analisando os gráficos de dados destes eventos e classificando-os, segundo os perfis dos choques feitos por E. Echer et al. [2003], em choques frontais, rápidos e lentos; ou reversos, rápidos e lentos. Este estudo deteve-se ao período de 2002 - 2003, que corresponde ao declínio do Ciclo Solar 23.

\section{Introdução}

O meio interplanetário está permeado pelo vento solar que é resultado de uma enorme diferença de pressão de gás entre a Corona Solar e o Espaço Interestelar. Esta diferença conduz o plasma para fora, apesar da influência da gravidade solar. A comunidade científica possui interesse em estudar o vento solar, principalmente nas Relações Sol-Terra, como mostra na Figura 1, pois este distante e tênue plasma é significantemente influenciado pela atividade solar e transmite esta influência para os planetas, cometas, partículas e raios cósmicos que nele estão imersos. A origem da influência

solar através da interação do campo magnético solar com o plasma coronal tem sido o maior tópico da pesquisa do vento solar.

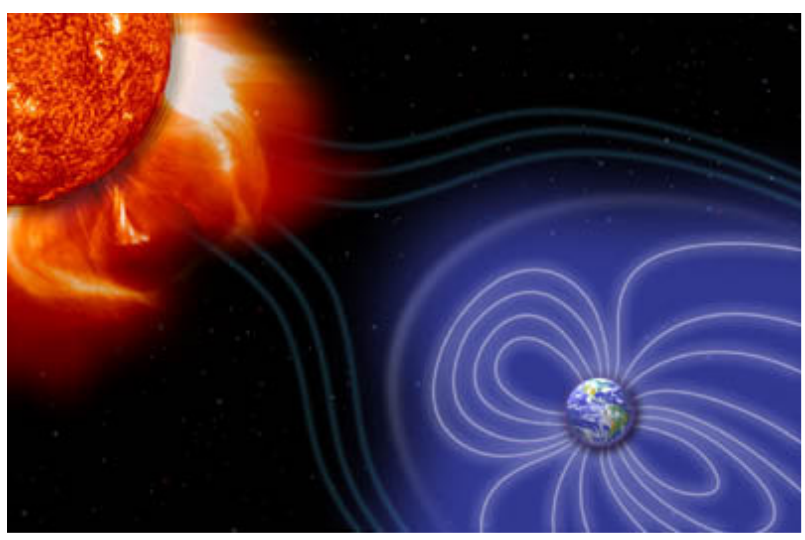

Figura 1 - Interações Sol - Terra principal interesse no estudo do vento solar. Fonte: http://www.spaceweathercenter.org

O vento solar que passa pelas proximidades da Terra é constituído principalmente por hidrogênio ionizado, com uma pequena mistura de hélio ionizado ( $5 \%)$, e íons de elementos mais pesados. Embebido neste plasma está um campo magnético com orientação aproximadamente paralela ao plano da Eclíptica, mas em aproximadamente $45^{\circ}$ à linha Sol-Terra para um observador a 1UA (Uma Unidade Astronômica, $1,49598 \times 10^{8} \mathrm{Km}$ ). O espaço interplanetário apresenta uma densidade extremamente baixa (cerca de cinco átomos por $\mathrm{cm}^{3}$ ), sendo um meio não colisional. Neste meio, o empilhamento de ondas não lineares pode ocorrer e gerar choques. $O$ vento solar favorece a ação das forças Coulombianas de longo alcance. Essas forças exercem no meio interplanetário, um papel semelhante ao das colisões. Colisões em um gás ordinário servem para transferir momentum e energia entre as moléculas, além de fornecer o acoplamento para que a onda sonora, meio básico de transferência de informação, exista (Burgess, 1995). Em um plasma sem colisões, tal acoplamento colisional está ausente, pois o livre caminho médio entre as colisões é muito maior que 
o sistema (E. Echer, tese, 2005). No caso do plasma interplanetário, o livre caminho médio na órbita da Terra é da ordem da distância Terra-Sol.

Além do empilhamento das ondas não lineares outros fatores podem produzir ondas de choques, por exemplo, as regiões de interação corrotante (RIC ou RI), quando a compressão do vento solar mais lento é feita pelos feixes rápidos emitidos pelos buracos coronais, provoca 0 desenvolvimento de choque, principalmente do tipo reverso a $1 \mathrm{UA}$, e mais raramente frontal rápido (Burlaga, 1995); enormes ejeções de material coronal expelidas do Sol, que se propaga no meio interplanetário, EMCls, a maioria das ondas de choque detectadas próximo à órbita da Terra, são causadas por EMCls. (Gonsalez et al. 1999).

Choques interplanetários podem ser classificados de acordo com a propagação relativa ao Sol, se estiver se propagando para o Sol é dito choque reverso ou para longe do Sol é dito choque frontal; mas podem ser classificados de acordo com o modo de onda, se a velocidade relativa ao vento solar é maior que o modo rápido da onda magnetossônica, velocidade característica do meio, é considerado choque rápido ou se é maior que o modo lento da onda magnetossônica é considerado choque lento. (Echer et al. 2003).

Na Figura 2, podemos observar os perfis dos parâmetros densidade (Np), temperatura (Tp), e velocidade (VSW) de próton e campo magnético $(B)$, para diferentes tipos de choque. Em choques frontais rápidos observam-se saltos abruptos positivos em todos os parâmetros. Em choques frontais lentos há um salto abrupto negativo no parâmetro $\mathrm{B}$; em choques reversos há saltos negativos nos parâmetros de Tp e Np e saltos positivos em VSW, e se for um choque do tipo rápido, há saltos abruptos negativos em $\mathrm{B}$ e, caso for lentos há um salto positivo em B.

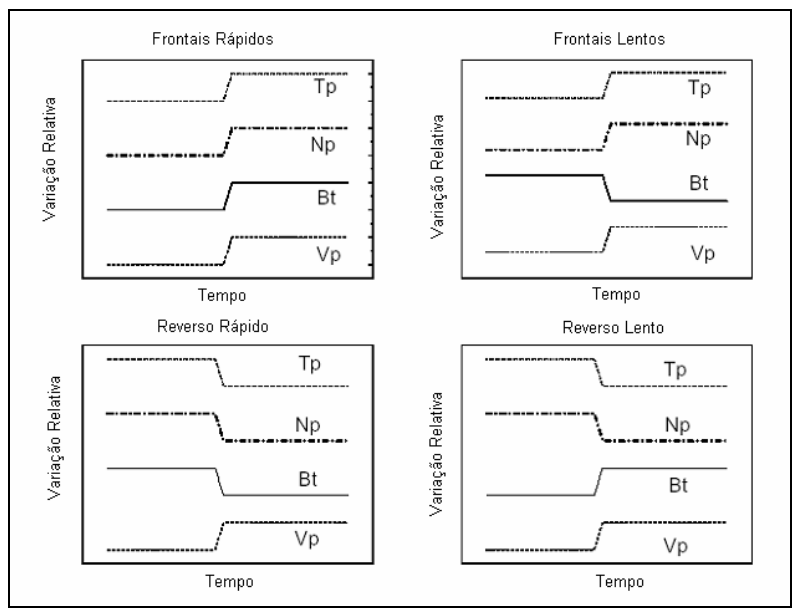

Figura 2 - Diagrama ilustrando os perfis de quatro tipos diferentes de choques: Frontais Rápidos, Frontais Lentos, Reversos Rápidos e Reversos Lentos, com a respectiva variação nos parâmetros de plasma e campo magnético interplanetário. [Adaptado de Echer et al, 2003]

A dinâmica das ondas existentes no vento solar é chamada Magnetohidrodinâmica (MHD), devido ao fato dessas ondas estarem imersas num fluido, o plasma, que possui propriedades magnéticas. Um choque MHD perpendicular é aquele em que o campo magnético no lado upstream, precedente à chegada do fluxo causador do choque, é perpendicular à normal à frente de choque. A única onda que se propaga perpendicular a $\mathbf{B}$ é a onda magnetossônica. O fluxo entra com uma velocidade maior que a velocidade magnetossônica e sai com uma velocidade menor que a velocidade magnetossônica. Portanto 0 choque propaga-se supermagnetossonicamente. Para um choque ser considerado perpendicular a equação a seguir deve ser satisfeita:

$$
\frac{\overrightarrow{B_{d}}}{\overrightarrow{B_{u}}}=\frac{N p_{d}}{N p_{u}}
$$

onde, $\overrightarrow{\mathrm{B}}_{\mathrm{u}}$ e $\mathrm{Np}_{\mathrm{u}}$ são os valores da intensidade do campo magnético e densidade de prótons respectivamente upstream, $\vec{B}_{d}$ e $N p_{d}$ são os valores da intensidade do campo magnético e densidade de prótons respectivamente downstream, posterior ao fluxo causador do choque. Esta equação diz que a direção do campo magnético não muda através de um choque perpendicular.

\section{Material e métodos}

Utilizamos dados de plasma e CMI fornecidos pela sonda ACE - Advanced Composition Explorer disponíveis em: <http://www.srl.caltech.edu/ACE/ASC/level2/index.html> com resolução temporal de 64 segundos. $\mathrm{Na}$ análise das variações de parâmetros, dividimos os gráfico em 3 janelas distintas de 10 minutos cada, o intervalo de turbulência na chegada do choque (S), outra antes da chegada do fluxo (U) e uma última depois do fluxo (D). Fizemos um cálculo do valor médio de cada parâmetro e determinamos as variações dos parâmetros de plasma e campo magnético, e seus respectivos erros de desvio padrão da média,

$$
\sigma=\sqrt{\frac{1}{n-1} \sum_{i=1}^{n}\left(X_{i}-\bar{X}\right)^{2}}
$$

Onde $\mathrm{n}$ é o número de dados utilizados neste caso $10, \mathrm{X}$ é o valor da variável e $\bar{X}$ é a média aritmética entre os valores utilizados. No cálculo da incerteza da ortogonalidade do choque utilizamos a seguinte equação:

$$
\sigma_{r x}={ }_{-}^{+} r_{x} *\left(\frac{\sigma_{x u}}{x u}+\frac{\sigma_{x d}}{x d}\right)
$$

onde $r_{x}$ é a razão entre duas grandezas, Np ou B, XU e $x d$, são os valores de uma grandezas, respectivamente antes e depois do choque, $\sigma_{x u}$ e $\sigma_{x d}$ são os erros dos valores médios, respectivamente antes e depois do choque. Posteriormente calculamos a intensidade de cada evento com as equações de Rankine-Hugoniot. Estas equações são relações físicas fundamentais para uma superfície plana de descontinuidade (choque), através da qual há um salto nos campos físicos dos upstream para 0 downstream. Essas equações expressam a conservação da massa, de fluxo de 
momentum tangencial, de energia e de fluxo magnético. Burlaga (1995) apresenta estas equações relativamente a um sistema de referência com origem no choque.

Se um choque esta se movendo radialmente para longe do Sol com velocidade $U_{s}$ relativa ao Sol, sendo as velocidades de plasma nos lados upstream e downstream respectivamente $u_{1}$ e $u_{2} \quad e$ predominantemente radiais, então a conservação de massa fornece a velocidade do choque em termos da densidade e da velocidade medidas por uma única espaçonave:

$$
U_{s}=\frac{n_{2} u_{2}-n_{1} u_{1}}{n_{2}-n_{1}}
$$

Onde, $\mathrm{n}_{1}$ e $\mathrm{n}_{2}$ são respectivamente as densidades upstream e downstream.

\section{Exemplos}

Neste trabalho estudamos detalhadamente cada tipo de choque e as figuras seguintes mostram exemplos de eventos estudados. O inicio do choque é indicado por uma linha nas figuras a seguir.

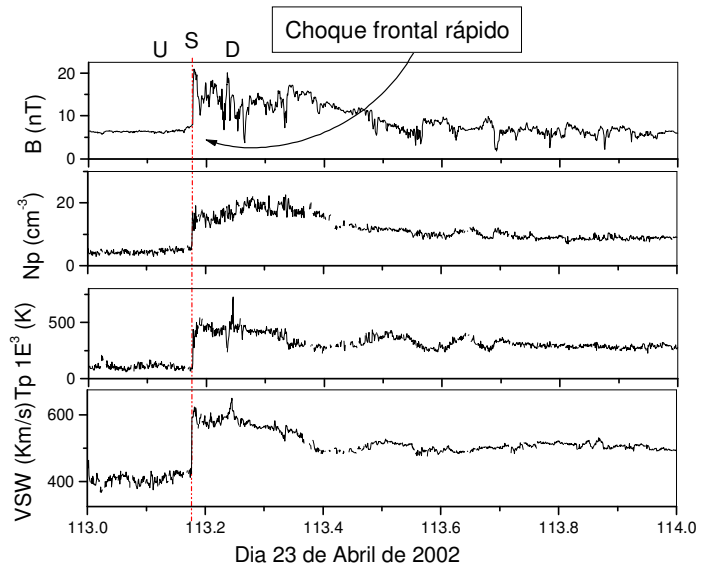

Figura 3 - Exemplo de um choque frontal rápido do dia 23 de abril de 2002. Onde houvera saltos abruptos em todos os parâmetros de plasma densidade (Np), temperatura (Tp), velocidade (VSW) e campo magnético interplanetário (B).

A Figura 3 é um exemplo de choque que está se movendo para fora do Sol em relação ao Sol, portanto, frontal, e rápido por sua velocidade relativa ao vento solar ser maior que o modo rápido da onda magnetossônica. Pode-se observar com clareza a chegada do fluxo e os saltos nos parâmetros nos lados upstream e downstream. É apresentado na Figura 4, um exemplo de um choque Reverso, ou seja, está se propagando para o Sol com relação ao mesmo e Rápido, pois a velocidade relativa ao vento solar é maior que o modo rápido da onda magnetossônica.

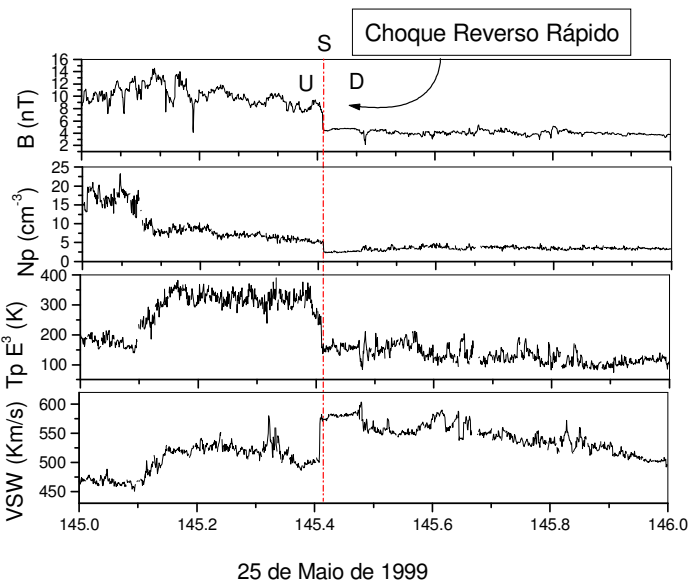

Figura 4 - Exemplo de um choque reverso rápido onde há saltos abruptos negativos nos parâmetros de temperatura (Tp), densidade (Np) e campo magnético (B) e saltos abruptos positivos no parâmetro de velocidade do vento solar (VSW).

\section{Resultados}

Uma análise das porcentagens de cada perfil de choque feita com o total de eventos ocorridos no período de 2002-2003 é apresentada na Figura 5. Foram analisados 83 choques deste período, deste total, 27 ocorreram no ano 2003 e 56 no ano 2002.

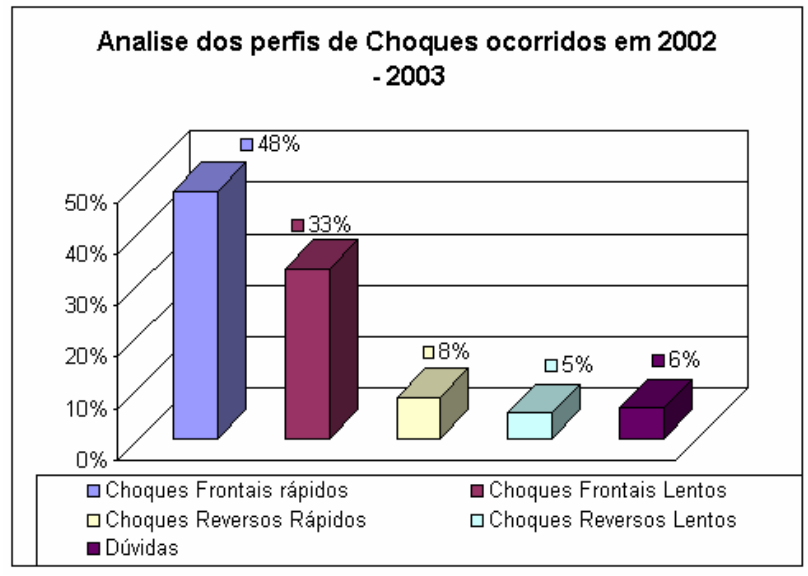

Figura 5 - Diagrama representando as percentagens dos perfis dos choques ocorridos em 2002 - 2003.

Cálculos de velocidade e variação de parâmetros de eventos ocorridos no período de 2002 - 2003 exemplificando cada tipo diferente de choque são mostrados na Tabela 1. Além do cálculo das variações de cada parâmetro foram calculadas: a velocidade Us de cada choque e as razões: 


$$
\begin{gathered}
r B=\frac{B d}{B u} \\
r N p=\frac{N p d}{N p u}
\end{gathered}
$$

Estes últimos cálculos são importantes para sabermos se a direção do choque é ou não perpendicular, através da relação (1).

Tabela 1 - Exemplo do cálculo da variação de parâmetros para cada tipo de choque onde, os subscritos, $u$ e d, representam respectivamente upstream e downstream. Us representa a velocidade do choque medidas por uma única espaçonave, e $r \mathrm{~B}$ e $r \mathrm{~Np}$

\begin{tabular}{|c|c|c|c|c|}
\hline \multicolumn{5}{|c|}{ Exemplos de cálculo de parâmetros } \\
\hline & $\begin{array}{c}\text { Choque Reverso } \\
\text { Rápido }\end{array}$ & $\begin{array}{c}\text { Choque Reverso } \\
\text { Lento }\end{array}$ & $\begin{array}{c}\text { Choque Frontal } \\
\text { Rápido }\end{array}$ & $\begin{array}{c}\text { Choque Frontal } \\
\text { Lento }\end{array}$ \\
\hline & 06/02/2002 02:03 UT & 03/03/2002 22:00 UT & 23/04/2002 04:15 UT & 14/10/2003 16:22 UT \\
\hline $\mathrm{Npu}\left(/ \mathrm{cm}^{3}\right)$ & $8,6_{-}^{+} 0,64$ & $41_{-}^{+} 1,62$ & $5,76_{-}^{+} 0,52$ & $15,52_{-}^{+} 0,56$ \\
\hline $\operatorname{Npd}\left(/ \mathrm{cm}^{3}\right)$ & $5,5_{-}^{+} 0,67$ & $20_{-}^{+} 1,21$ & $15,25_{-}^{+} 2,02$ & $21,04_{-}^{+} 1,52$ \\
\hline$\Delta \mathrm{Np}\left(\mathrm{lcm}^{3}\right)$ & $-3,1$ & $-21,6$ & 9,50 & 5,52 \\
\hline Tpu $(\mathrm{K})$ & 310522 & 35985,1 & 85864,83 & 90116,86 \\
\hline Tpd $(\mathrm{K})$ & 248,342 & 26248,3 & 439258,89 & 212890,00 \\
\hline$\Delta \mathrm{Tp}(\mathrm{K})$ & $-62,180$ & $-9,737$ & 353394,06 & 122773,14 \\
\hline VSWu $(\mathrm{Km} / \mathrm{s})$ & 624,8 & 353,1 & 430,11 & 481,89 \\
\hline $\operatorname{VSWd}(\mathrm{km} / \mathrm{s})$ & 650,9 & 367,9 & 599,78 & 516,66 \\
\hline$\Delta \mathrm{VSW}(\mathrm{Km} / \mathrm{s})$ & 26,1 & 14,8 & 169,67 & 34,76 \\
\hline $\mathrm{Bu}(\mathrm{nT})$ & $14,5_{-}^{+} 0,33$ & $10,1_{-}^{+} 0,35$ & $7,42_{-}^{+} 0,11$ & $15,64_{-}^{+} 0,18$ \\
\hline $\mathrm{Bd}(\mathrm{nT})$ & $8,8_{-}^{+} 0,18$ & $15,1_{-}^{+} 0,17$ & $16,80_{-}^{+} 2,84$ & $8,53_{-}^{+} 2,02$ \\
\hline$\Delta \mathrm{B}(\mathrm{nT})$ & $-5,7$ & 5,1 & 9,38 & $-7,10$ \\
\hline Us $(\mathrm{Km} / \mathrm{s})$ & 578,5 & 115,8 & 702,61 & 614,41 \\
\hline rB & $0,61{ }_{-}^{+} 0,03$ & $1,51_{-}^{+} 0,07$ & $2,26{ }_{-}^{+} 0,42$ & $0,55_{-}^{+} 0,14$ \\
\hline $\mathrm{rNp}$ & $0,64_{-}^{+} 0,12$ & $0,48_{-}^{+} 0,05$ & $2,64_{-}^{+} 0,58$ & $1,36_{-}^{+} 0,15$ \\
\hline \multicolumn{5}{|c|}{ Vetores em coordenadas GSE } \\
\hline $\mathrm{Vu}(\mathrm{Km} / \mathrm{s})$ & {$[-615.9 ; 95.1 ;-32.5]$} & {$[-346.9 ;-56.5 ;-33.1]$} & {$[-428.51 ;-9.59 ; 34.26]$} & {$[-477.97 ;-59.30 ;-15.40]$} \\
\hline $\mathrm{Vd}(\mathrm{km} / \mathrm{s})$ & {$[-648.1 ; 55.5 ; 1.9]$} & {$[-364.2 ;-45.2 ;-25.2]$} & {$[-597.23 ; 30.41 ; 5.15]$} & {$[-514.90 ;-38.63 ; 1.60]$} \\
\hline $\mathrm{Bu}(\mathrm{nT})$ & {$[-9.9 ; 9.2 ; 3.7]$} & {$[6.4 ;-4.1 ; 6.2]$} & {$[-1.56 ; 4.14 ;-5.56]$} & {$[-10.53 ; 8.38 ; 7.86]$} \\
\hline $\mathrm{Bd}(\mathrm{nT})$ & {$[-60.4 ; 50.9 ;-0.9]$} & {$[-7.9 ; 12.5 ;-1.6]$} & {$[-0.05 ; 9.06 ; 6.27]$} & {$[-1.02 ; 3.77 ;-3.30]$} \\
\hline
\end{tabular}
representam as razões entre os valores downstream e upstream da intensidade do campo magnético e da densidade respectivamente.

\section{Conclusões}

Neste trabalho apresenta-se uma breve revisão sobre conceitos básicos de vento solar, ondas de choque no meio interplanetário e suas possíveis origens.

Faz-se um estudos dos perfis dos choques interplanetários e com 0 auxilio das equações de Rankine-Hugoniot, calcula-se a velocidade Us de cada choque, além das razões entre as densidades e os campos magnéticos. Considerando a margem de erro das medidas, tem-se no dia 06/02/2002 um choque perpendicular, pois $r \quad B=0,61{ }_{-}^{+} 0,03$ que é aproximadamente igual ao valor de $r \mathrm{~Np}=0,64_{-}^{+} 0,12$, assim como no dia 23/04/2002 tem-se choque perpendicular, $r \mathrm{~B}=2,26_{-}^{+} 0,42$ que é aproximadamente igual ao valor de $\mathrm{rNp}=2,64_{-}^{+} 0,58$.

No estudo estatístico feito dos choques ocorridos no período de 2002 - 2003, foram analisados 83 eventos, 40 choques Frontais Rápidos; 5 Frontais Lentos; 7 Reversos Rápidos; 4 Reversos Lentos e 27 eventos, analisando somente os gráficos desses parâmetros e utilizando os critérios de análise mencionados no texto, não permitiram a identificação do tipo de choque. Assim é necessário estudar outros critérios/métodos que possam ser utilizados na classificação dos choques.

Conclui-se que a maioria dos choques que ocorreram no meio interplanetário no período de 2002 - 2003, foram do tipo Frontais Rápidos. Em trabalhos futuros estudaremos o motivo da predominância de choques frontais rápidos, se há ou não influência do declínio do ciclo solar, bem 
como elaborar uma análise comparativa entre os choques frontais e reversos.

\section{Agradecimentos}

Os autores gostariam de agradecer ao programa $\mathrm{PIBIC/CNPq}$ pelo financiamento de bolsas de Iniciação Científica, processos 119579/2004-9 e 107615/2003-7, as quais são de grande importância para a realização de trabalhos científicos e ao desenvolvimento de mentes investigativas que contribuirão para 0 aumento da comunidade científica futura.

Os autores gostariam de agradecer a missão ACE pela disponibilidade dos dados de plasma e CMI utilizados neste trabalho.

\section{Referências}

Burgess, D., Colissionless shocks, chapter 5, in MG Kivelson and CT Russell (eds.), Introduction to Space Physics, Cambridge University Press, Cambridge, 1995.
Burlaga,
L.F.,
1995.
Interplanetary

Magnetohydrodynamics. Oxford University Press, New

York

Echer, E., W. D. Gonzalez, L. E. A. Vieira, A. Dal Lago, F. L Guarnieri, A. Prestes, A. L C. Gonzalez and N. J. Schuch, Interplanetary shock parameters during solar activity maximum (2000) and minimum (1995-1996), Brazilian Journal of Physics, 33, 115-122, 2003.

Gonzalez, W. D., B. T. Tsurutani, and A. L. Cla de Gonzalez, Space Science Reviews 88, 529 (1999). 